\title{
Proteção farmacológica da medula espinal isquêmica
}

\author{
Albert Amin SADER*, Joaquim COUTINHO NETTO*, João José LACHAT*, \\ José Eduardo de Salles ROSELINO*, Flávio José BALLERINI*
}

RBCCV 44205-296

\begin{abstract}
Sader A A, Coutinho Netto J, Lachat J J, Roselino J E S, Ballerini F J - Proteção farmacológica da medula espinal isquêmica. Rev Bras Cir Cardiovasc 1996; 11 (2): 96-106.

RESUMO: Estudou-se a ação protetora da quetamina ( $30 \mathrm{mg} / \mathrm{kg}, E V)$ e da clorpromazina ( $2 \mathrm{mg} / \mathrm{kg}, E V)$, sobre a medula espinal de ratos Wistar, submetida à isquemia de $30 \mathrm{~min}$, por oclusão da aorta torácica, seguida de reperfusāo. Em 70 animais, com peso médio de $380 \mathrm{~g}$, divididos em 7 grupos iguais, obtiveram-se os seguintes resultados porcentuais referentes à integral recuperação sensitivo-motora: 1) "Sham-operation": $100 \% ; 2$ ) isquemia-reperfusão: $0 \%$; 3) quetamina, 1 min antes da isquemia: $30 \% ; 4$ ) quetamina, 10 min antes da isquemia: $50 \% ; 5$ ) clorpromazina, 1 min antes da isquemia: $50 \% ; 6$ ) clorpromazina, 1 min antes da reperfusão: $10 \%$; 7) quetamina + clorpromazina, 1 min antes da isquemia: $60 \%$. Tanto a quetamina quanto a clorpromazina protegeram parte dos animais cuja medula espinal fora submetida à isquemia-reperfusão. Contudo, ao se comparar os animais protegidos, as diferenças de resultados só alcançaram significância estatística entre os grupos 6 e 7. O estudo histológico, por microscopia óptica, confirmou a açāo protetora de ambos os agentes farmacológicos. A perfusāo do espaço subaracnóideo dos animais cuja medula espinal fora submetida à isquemia-reperfusāo demonstrou quantidade excessiva dos aminoácidos neuroexcitadores, Laspartato e L-glutamato.
\end{abstract}

DESCRITORES: Medula espinal, efeito de drogas. Isquemia, fisiopatologia. Quetamina, farmacologia. Clorpromazina, farmacologia. Medula espinal, isquemia.

\section{INTRODUÇÃO}

A extrema sensibilidade das células neuronais do sistema nervoso central (SNC) à anóxia e à isquemia é um fato perfeitamente conhecido e balizador de numerosas condutas e procedimentos médicos. Os cirurgiōes cardiovasculares têm que se preocupar, cotidianamente, com a integridade do encéfalo e da medula espinal durante as operações realizadas no coração e na aorta.

Indiscutivelmente, constitui uma ameaça constante no per-operatório e tem sido um dos principais fatores limitantes do desenvolvimento das operações cardiovasculares. Com efeito, esse tipo de operação só se efetivou a partir do momento em que os cirurgiões encontraram meios de proteger 0 SNC durante as intervençōes no coração e na aorta. A hipotermia, sistêmica ou regional (1-9), e a circulação extracorpórea (10-12), isoladas ou associadas, são os recursos usados desde o início da década de 50. A primeira, reduzindo a atividade metabólica $e$, conseqüentemente, a demanda de $\mathrm{O}_{2}$; a segunda, garantindo o fluxo sangüineo e a oferta de $\mathrm{O}_{2}$ e outros metabólitos, sobretudo a glicose.

Trabalho realizado nos Departamentos de Cirurgia, Ortopedia e Traumatologia (Laboratório de Cirurgia Experimental), Bioquimica e Morfologia da Faculdade de Medicina de Ribeirăo Preto da Universidade de São Paulo. Ribeirão Preto, SP, Brasil. 
Sader A A, Coutinho Netto J, Lachat J J, Roselino J ES, Ballerini F J - Proteção farmacológica da medula espinal isquêmica. Rev Bras Cir Cardiovasc 1996; 11 (2): 96-106.

Contudo, há situações em que a interrupção do fluxo sangüíneo a regiōes do SNC é inevitável ou desejável sob o aspecto da estratégia e da tática cirurgicas, razão pela qual o desenvolvimento de meios de proteção aos neurônios isquêmicos é altamente desejável.

O gradiente entre a pressão arterial média (PAM) e a pressão liquórica representa a pressão efetiva de perfusão do SNC $(13,14)$. O pinçamento da aorta torácica descendente determina drástica redução da PAM, distalmente, e, por conseqüência, do fluxo sangüíneo da medula espinal, sobretudo na transição toracoabdominal, dependente também do variável padrão anatômico da rede vascular arterial (1518). A drenagem liquórica, reduzindo a pressão no espaço subaracnóideo, tende a manter o referido gradiente, conferindo proteção à medula espinal isquêmica $(10,19-22)$

Ao nivel celular, as diversas etapas dos mecanismos de lesão causados pela isquemia e isquemia com reperfusão têm se mostrado uniformes e consistentes, independente do tecido onde ocorre. Certos tecidos são mais susceptíveis à isquemia que outros, estando as células excitáveis do coração e do SNC entre as mais vulneráveis. A vulnerabilidade seletiva, e conseqüente morte rápida ou tardia dos neurônios do SNC, deve-se à agressão adicional do aumento da concentração dos aminoácidos excitadores (AAE), L-glutamato e L-aspartato.

A proteção farmalógica da medula espinal isquêmica tem sido alcançada, em trabalhos experimentais, com o emprego de numerosos agentes, isolada ou associadamente, alguns dos quais em uso na prática médica: bloqueadores dos canais de cálcio $(14,23-26)$; removedores de radicais livres de $\mathrm{O}_{2}$ (26-30); moduladores do sistema imunológico (31, 32); antagonistas dos aminoácidos neuroexcitadores (33-35); drogas anestésicas e similares (36-43); corticosteróides (22).

No presente trabalho, avaliou-se a ação da quetamina e da clorpromazina, como agentes farmacológicos protetores da medula espinal de ratos, submetida a isquemia e reperfusão. O primeiro, conhecido anestésico e antagonista não competitivo do receptor do L-glutamato, tipo $\mathrm{N}$-metil-D-aspartato, (NMDA), quando administrado previamente, revelouse capaz de proteger as estruturas nervosas da retina isquêmica de coelhos,com ou sem reperfusão $(44,45)$, e da medula espinal isquêmica de coelhos (43). $O$ segundo, uma fenotiazina com ações múltiplas, tais como vasodilatadora e inibidora de fosfolipases, entre outras, tem sido eficaz na prevenção das lesões de isquemia e reperfusão do fígado $(46,47)$; coração $(48-50)$ e sistema nervoso central $(51,52)$.

\section{MATERIAL E MÉTODOS}

\section{1- Animais}

Em todos os experimentos usaram-se ratos Wistar, pesando entre $325 \mathrm{~g}$ e $510 \mathrm{~g}$ (média $380 \mathrm{~g}$ ), convenientemente agrupados.

\section{II - Isquemia-Reperfusão da Medula Espinal e Proteção Farmacológica}

Setenta ratos formaram 7 grupos de igual número. Todos foram anestesiados mediante indução com éter sulfúrico inalado dentro de uma campânula, seguindo-se intubação orotraqueal com uma cânula preparada com um tubo de Polietileno (PE - 350). Através dela, manteve-se a anestesia até o final do procedimento, com uma mistura de éter e clorofórmio, em partes iguais, vaporizada pela passagem de oxigênio. Usou-se, para isso, o vaporizador e o respirador de Takaoka mod 600. A intensidade da vaporização variou na dependência das necessidades para manter os animais em plano adequado de anestesia; nos que receberam quetamina e/ou clorpromazina foi reduzida e, às vezes, descotinuada.

Em todos os animais fez-se toracotomia lateral esquerda no quarto espaço intercostal, fechada no final do experimento com os cuidados necessários à completa reexpansão pulmonar. Após a reversão anestésica, retirou-se o tubo orotraqueal e, no pósoperatório, os animais foram cuidadosamente observados quanto à recuperação sensitivo-motora das patas posteriores e cauda.

\section{Grupos de Tratamento}

Grupo 1 (Controle-"Sham operation") - A aorta torácica imediatamente distal à emergência da artéria subclávia esquerda foi isolada e envolvida pelo garrote, mas não ocluída, por 30 minutos.

Grupo 2 (Isquemia-reperfusão) - A aorta torácica foi manipulada como no Grupo 1 , com a única diferença de ter sido ocluída completamente durante 30 minutos, seguindo-se reperfusão.

Grupo 3 (Queratina 1) - Operados como os do Grupo 2, os animais receberam, 1 minuto antes do garroteamento aórtico, cloridrato de quetamina injetado por via endovenenosa (EV), na dose de 30 $\mathrm{mg} / \mathrm{kg}$ de peso corpóreo.

Grupo 4 (Quetamina 2) - Neste grupo, o cloridrato de quetamina foi injetado, na mesma dose, 10 minutos antes da oclusão aórtica.

Grupo 5 (Clorpromazina 1) _ Os animais rece- 
Sader A A, Coutinho Netto J, Lachat J J, Roselino J ES, Ballerini F J - Proteção farmacológica da medula espinal isquêmica. Rev Bras Cir Cardiovasc 1996; 11 (2): 96-106.

beram clorpromazina EV na dose de $2 \mathrm{mg} / \mathrm{kg}$ peso corpóreo, 1 minuto antes da oclusão aórtica.

Grupo 6 (Clorpromazina 2) - Neste grupo, a clorpromazina foi administrada, na mesma dose, 1 minuto antes da reperfusão aórtica.

Grupo 7 (Quetamina + Clorpromazina) - Os ratos receberam clorpromazina EV (2 $\mathrm{mg} / \mathrm{kg}$ peso) e quetamina EV (30 mg/kg peso) 1 minuto antes da oclusão aórtica.

Os dados principais referentes aos animais dos 7 Grupos encontram-se resumidos na Tabela 1.

Para a análise estatística, aplicou-se o teste exato de Fisher.

\section{III - Estudo Histológico}

Para o estudo histológico da medula espinal foram usados 3 animais dos Grupos 3, 4, 5 e 7, que recuperaram a função motora e a sensibilidade. Para o estudo dos Grupos 1 e 2, fizeram-se 3 experimentos adicionais, especificamente para esse fim.

Os animais foram profundamente anestesiados com éter sulfúrico, heparinizados, e, através de cânula introduzida pela ponta do ventrículo esquerdo, fez-se a perfusão do sistema arterial, antes com solução de $\mathrm{NaCL} 0,9 \%$ e, depois, com formol $10 \%$.

Após a perfusão, a medula espinal foi retirada e os seus segmentos cervical, torácico, lombar e sacral foram separados e colocados no mesmo fixador por 72 horas e então processados para inclusão em parafina.

Fizeram-se cortes com 5 micrômetros de espessura de cada um dos segmentos medulares, corados com hematoxilina e eosina e analisados microscopicamente.

\section{IV - Perfusão da Medula Espinal Isquêmica e Analise de Aminoácidos}

A anestesia foi induzida em 6 ratos, por injeção intraperitoneal de tiopental sódico (Abbott), $40 \mathrm{mg} /$ $\mathrm{kg}$ de peso corpóreo. A aorta abdominal de cada rato foi exposta proximalmente às artérias renais $(0,5 \mathrm{~cm})$ e envolvida por um garrote. Suturou-se a parede abdominal, posicionando-se o rato em decúbito ventral para o implante da cânula de perfusão. Após incisão ao nível de S3-S4, expôs-se delicadamente os processos dessas vértebras com afastamento e exérese dos músculos contíguos, seguindo-se com a laminectomia por intermédio de uma broca carborundum tronco-cônica. Para o implante no espaço subaracnóideo, utilizou-se uma cânula tipo push-pull, confeccionada pela inserção de uma agulha Mizzi no interior de um tubo de Polietileno PE-10. Manteve-se a cânula posicionada no canal medular com cimento cianoacrilato, misturado com fosfato de zinco. Para a perfusão utilizou-se solução de cloreto de sódio $0,85 \%$, em fluxo contínuo de 7,0 $\mu 1 /$ minuto, com bomba de microperfusão, sendo o perfusato colhido em tubos contendo $0,10 \mathrm{ml}$ de $\mathrm{HCl}$ $0,5 \mathrm{~N}$. Após estabilização de 40 minutos, 6 amostras controles foram coletadas a cada 5 minutos, executando-se a seguir o garroteamento completo da aorta por 40 minutos, seguindo-se um período de 30 minutos de reperfusão.

Os aminoácidos recolhidos no perfusato, préderivatizados com ortoftalaldeído (OPA ), foram analisados em HPLC Shimadzu, utilizando-se coluna Supersphere $5 \mu \mathrm{m}$, ODS-C18 (Merck) e detecção fluorescente pelo método de LINDROTH \& MOPPER ${ }^{(53)}$.

TABELA 1

PRINCIPAIS DADOS E AVALIACĀO CLÍNICA DOS RATOS CUJA MEDULA ESPINAL, SUBMETIDA A ISQUEMIA E REPERFUSĀO, FOI PROTEGIDA OU NÃO POR AGENTES FARMACOLÓGICOS

\begin{tabular}{|c|c|c|c|c|}
\hline GRUPO & $\begin{array}{c}\text { AGENTE } \\
\text { FARMACOLÓGICO }\end{array}$ & $\begin{array}{l}\text { DOSE } \\
M G / K G\end{array}$ & $\begin{array}{c}\text { MOMENTO DA } \\
\text { ADMINISTRAÇÃ̄O }\end{array}$ & $\begin{array}{l}\text { RECUPERAÇÃO } \\
\text { MOTORA E } \\
\text { SENSITIVA (\%) }\end{array}$ \\
\hline $1(\mathrm{~N}=10)$ & $\begin{array}{l}\text { "Sham } \\
\text { operation" }\end{array}$ & - & - & 100 \\
\hline $2(\mathrm{~N}=10)$ & $\begin{array}{l}\text { Isquemia } \\
\text { reperfusão }\end{array}$ & - & - & 0 \\
\hline $3(N=10)$ & Quetamina & 30 & 1 min pré-isquemia & 30 \\
\hline $4(\mathrm{~N}=10)$ & Quetamina & 30 & 10 min pré-isquemia & 50 \\
\hline $5(\mathrm{~N}=10)$ & Clorpromazina & 2 & $1 \mathrm{~min}$ pré-isquemia & $(4 \mid x)$ \\
\hline $6(N=10)$ & Clorpromazina & 2 & 1 min pré-reperfusão & 10 \\
\hline $7(\mathrm{~N}=10)$ & $\begin{array}{l}\text { Quetamina } \\
+ \\
\text { Clorpromazina }\end{array}$ & $\begin{array}{r}30 \\
2\end{array}$ & 1 min pré-isquemia & 60 \\
\hline
\end{tabular}


Sader A A, Coutinho Netto J, Lachat J J, Roselino JES, Ballerini F J-Proteção farmacológica da medula espinal isquêmica. Rev Bras Cir Cardiovasc 1996; 11 (2): 96-106.

\section{RESULTADOS}

Avaliação Clínica dos Animais Submetidos ou não à Proteção Farmacológica e Seus Controles.

Grupo 1 (Controle-"Sham operation") - Todos os animais sobreviveram ao procedimento, recuperando a sensibilidade e os movimentos normais durante o periodo de obsrvação de 7 dias.

Grupo 2 (Isquemia-reperfusão) - Todos os animais deste grupo recuperaram-se da anestesia, com paralisia e anestesia das patas posteriores e cauda. Assim permaneceram até a morte, que ocorreu dentro de 6 horas.

Grupo 3 (Quetamina 1) - Três $(30 \%)$ animais recuperaram-se da anestesia e voltaram a caminhar com alguma dificuldade inicial. Nos dias subseqüentes, a motricidade e a sensibilidade das patas posteriores e cauda pareciam normais. Quatro $(40 \%)$ ratos não se recuperaram satisfatoriamente da anestesia e morreram dentro de 6 horas. Os demais $(30 \%)$ recuperaram-se da anestesia, paraplégicos, vindo a morrer depois de até 12 horas.

Grupo 4 (Quetamina 2) - Cinco $(50 \%)$ ratos recobraram os movimentos e a sensibilidade da cauda e patas posteriores, chegando a caminhar normalmente nos dias subseqüentes. Dois $(20 \%)$ animais recuperaram-se da anestesia paraplégicos, demonstrando pouca sensibilidade na cauda e patas posteriores. Outros $2(20 \%)$ estavam paraplégicos, sem qualquer sensibilidade, e $1(10 \%)$ não se recuperou da anestesia. Os animais que não recobraram a motricidade morreram dentro de 4 horas.

Grupo 5 (Clorpromazina 1) - Seis $(60 \%)$ ratos voltaram a se movimentar com as patas posteriores, um dos quais com dificuldade, vindo a morrer 24 horas depois. Os demais readquiriram movimentação e sensibilidade aparentemente normais. Os 4 $(40 \%)$ restantes morreram nas 24 horas subseqüentes, sem recuperar a motricidade e a sensibilidade.

Grupo 6 (Clorpromazina 2) - Apenas 1 (10\%) animal recobrou os movimentos e sensibilidade da cauda e patas posteriores. Um recuperou-se da anestesia paraplégico, porém com sensibilidade dolorosa da cauda, tendo sido sacrificado 48 horas depois. Um ficou paraplégico, demonstrando sensibilidade dolorosa da cauda, vindo a morrer 6 horas depois. Os 7 animais restantes recuperaram-se da anestesia paraplégicos, não demonstrando qualquer sensibilidade dolorosa da cauda e patas posteriores; morreram nas 7 horas subseqüentes.

Grupo 7 (Quetamina + Clorpromazina) - Seis $(60 \%)$ ratos recobraram os movimentos e sensibilidade dolorosa da cauda e patas posteriores, vol- tando a andar como antes. Três desses animais tiveram recuperação anestésica lenta. Dois $(20 \%)$ animais recuperaram-se da anestesia paraplégicos, e morreram nas 6 horas seguintes; outros $2(20 \%)$ morreram antes da reversão anestésica.

Os resultados da avaliação clínica, bem como os principais dados referentes aos vários grupos, encontram-se resumidos na Tabela 1.

Aplicando-se o teste exato de Fisher, foram obtidos os seguintes resultados: para a comparação dos grupos 3 e $4(P=0,4099) ; 3$ e $5(P=0,4099) ; 3$ e $6(P=0,5820) ; 3$ e $7(P=0,3698) ; 4$ e $6(P=0,0759)$; 4 e $7(P=1,0) ; 5$ e $6(P=0,0759) ; 5$ e $7(P=1,0) ; 6$ e $7(P=0,0302)$. Para $\alpha=0,05$, apenas o último resultado alcançou nível de significância estatística. Nas comparações feitas contra o grupo submetido a isquemia-reperfusão, tivemos os seguintes resultados: 2 e $3(P=0,1053) ; 2$ e $6(P=1,0)$. Todos os demais mostraram diferença significativa, isto é, 2 e $4(P=0,0163) ; 2$ e $5(P=0,0163) ; 2$ e $7(P=0,0054)$.

\section{Estudo Histológico}

O estudo dos cortes histológicos (Figura 1) mostrou que as medulas espinhais submetidas a isquemia e reperfusão (Grupo 2), principalmente nos niveis lombar e sacral, apresentam células nervosas do corno anterior com lesões graves, caracterizadas por edema intersticial, perda dos corpúsculos de Nissl e desestruturação dos núcleos. Nos animais dos grupos 4,5 e 7 , as lesões foram progressivamente menos graves e a medula espinhal apresentava uma estrutura próxima àquela observada nos controles (Grupo 1).

Perfusão e Analise Quantitativa dos Aminoácidos liberados da Medula Espinal Submetida a Isquemia e Reperfusão

As Figuras 2 e 3 mostram os resultados das análises cromatográficas dos AAE, L-glutamato e Laspartato, respectivamente, obtidos dos perfusatos liquóricos da medula espinal de ratos, coletados continuamente durante o período controle, período de isquemia medular por garroteamento da aorta abdominal durante 40 minutos e reperfusão medular após liberação do garrote. Ambos os AAE mostraram-se aumentados, significativa e especificamente, nos perfusatos liquóricos analisados durante todo o tempo de duração da isquemia, atingindo níveis máximos de $436 \%(5,33 \mu \mathrm{M})$ para o L-glutamato e $4673 \%(0,89 \mu \mathrm{M})$ para o L-aspartato, em relação aos níveis controles $(1,22 \mu \mathrm{M}$ para o L-glutamato $\mathrm{e}$ $0,02 \mu \mathrm{M}$ para o L-aspartato). Outros 15 aminoácidos isolados e quantificados, não monstraram alteração significativa. No período de reperfusão, somente o 
Sader A A, Coutinho Netto J, Lachat J J, Roselino JE S, Ballerini F J - Proteção farmacológica da medula espinal isquêmica. Rev Bras Cir Cardiovasc 1996; 11 (2): 96-106.
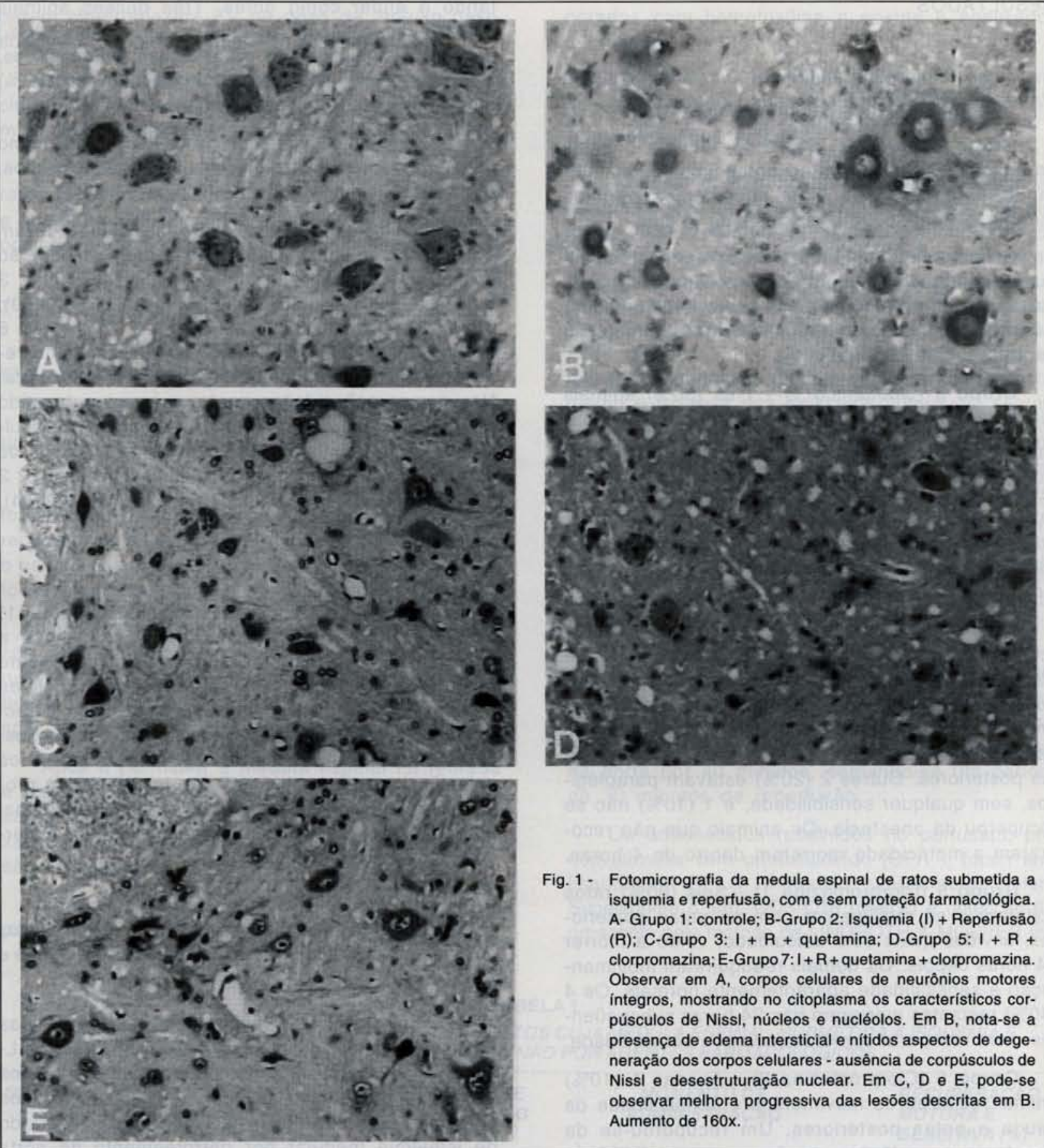

Fig. 1 - Fotomicrografia da medula espinal de ratos submetida a isquemia e reperfusāo, com e sem proteçāo farmacológica. A- Grupo 1: controle; B-Grupo 2: Isquemia (I) + Reperfusão (R); C-Grupo 3: I + R + quetamina; D-Grupo $5: 1+R+$ clorpromazina: E-Grupo 7: I+R + quetamina + clorpromazina. Observar em A, corpos celulares de neurônios motores integros, mostrando no citoplasma os característicos corpúsculos de Nissl, núcleos e nucléolos. Em B, nota-se a presença de edema intersticial e nítidos aspectos de degeneração dos corpos celulares - ausência de corpúsculos de Nissl e desestruturação nuclear. Em C, D e E, pode-se observar melhora progressiva das lesões descritas em B. Aumento de 160x.

L-aspartato mostrou-se significativamente aumentando (Figura 3).

\section{COMENTÁRIOS}

A lesão neuronal decorrente da isquemia depende da intensidade e duração do processo e se ocorre ou não hipóxia/anóxia (54-56). Inicia-se numa cascata de eventos bioquímicos, que têm como denominador comum o exagerado influxo intracelular de $\mathrm{Ca}^{++}{ }^{(57)}$. Desarranja-se o sistema enzimático (58) e as enzimas cálcio-ativadas, proteases, lipases e nucleases, são mediadoras de processos lesivos das células, comprometedores da síntese de proteínas (59). A formação exagerada e o acúmulo de radicais livres de $\mathrm{O}_{2}$ durante a reperfusão agravam as lesões isquêmicas (60). 
Sader A A, Coutinho Netto J, Lachat J J, Roselino JES, Ballerini F J - Proteção farmacológica da medula espinal isquêmica. Rev Bras Cir Cardiovasc 1996; 11 (2): 96-106.

\section{GRÁFICO 1}

ANÁLISE REPRESENTATIVA DA LIBERAÇÃO DE LGLUTAMATO NO LIQUORDAMEDULAESPINAL DERATO SUBMETIDA A ISQUEMIA E REPERFUSÄO

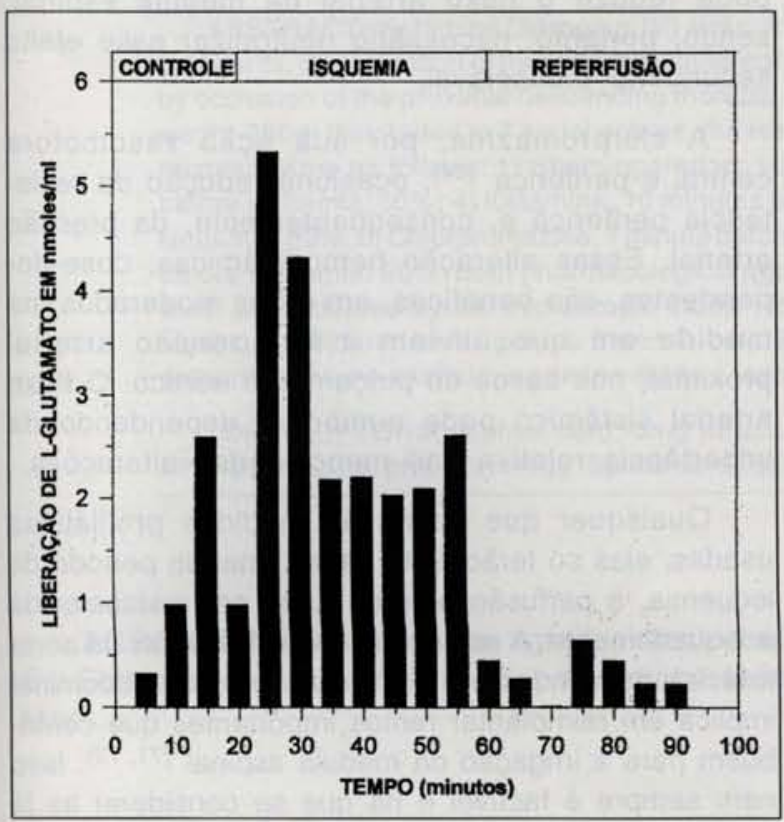

Um dos elos iniciais da cadeia é, certamente, a liberação excessiva de aminoácidos neuroexcitadores, glutamato e aspartato, os quais, hiperestimulando os receptores dos $\mathrm{AAE}$, principalmente o NMDA, ativam exageradamente os canais iônicos de $\mathrm{CA}^{++}$e $\mathrm{Na}^{+}$. Nas Figuras 2 e 3, observou-se aumento significativo e específico dos AAE, L-glutamato e L-aspartato, respectivamente, no perfusato liquórico da medula espinal durante o período isquêmico, atingindo concentração $>5,33 \mu \mathrm{M}$, nivel muito acima do necessário para provocar agressão causadora de morte neuronal rápida e seletiva (61).

Partindo desses conhecimentos e com o objetivo de prevenir ou reduzir, por intervenções farmacológicas, a magnitude das lesōes isquêmicas e de reperfusão ao SNC, dois caminhos principais têm sido seguidos: 1) bloqueios dos canais de $\mathrm{Ca}^{++}$ com drogas específicas $(14,23,25,57) ; 2)$ bloqueio dos receptores dos $\operatorname{AAE}, \operatorname{NMDA}(35,62)$ e não NMDA.

Das duas substâncias usadas nesta pesquisa, a clorpromazina já era conhecida por sua ação protetora dos hepatócitos isquêmicos $(46,47)$, do micárdio ${ }^{(48-50)}$ e do SNC $(51,52)$.

A maneira pela qual a clorpromazina, um derivado da fenotiazina, protege a célula isquêmica, provavelmente, obedece a multiplos mecanismos: retardando a ocorrência da despolarização anóxica (spreading depression), a qual está relacionada ao
GRÁFICO 2

ANÁLISE REPRESENTATIVA DO L-ASPARTATO LIBERADONOLIQUORDAMEDULAESPINALDERATOSUBMETIODA A ISQUEMIA E REPERFUSÃO

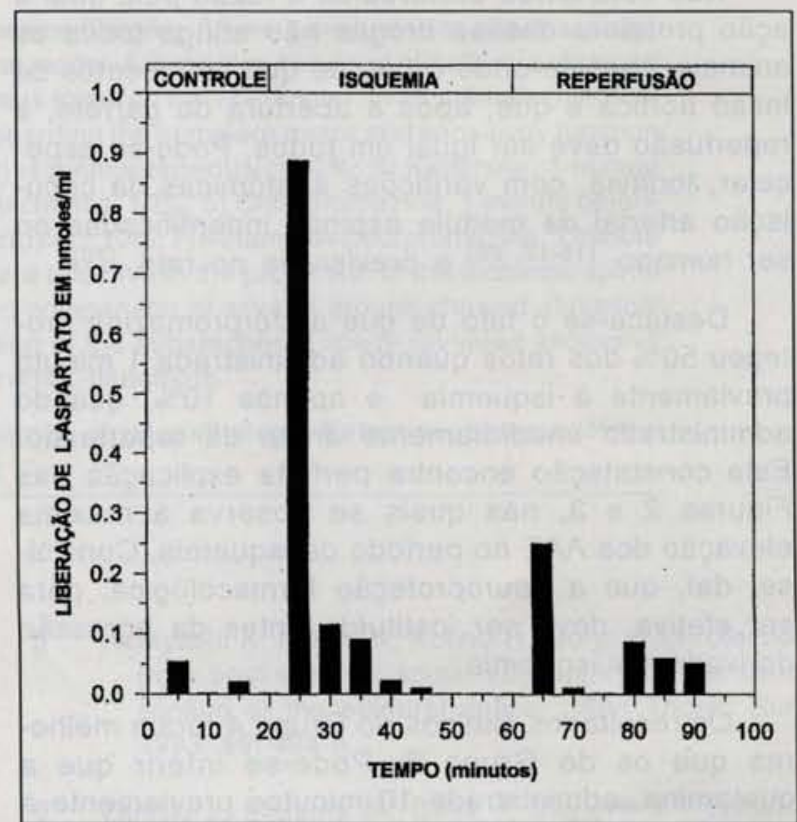

influxo de $\mathrm{Ca}^{++}$nos neurônios ${ }^{(51)}$; inibindo as kinases cálcio-dependentes (52); bloqueando receptores da dopamina (52); inibindo kinases calmodulina-dependentes $(52,63)$; por bloqueio dos canais de $\mathrm{Ca}++(46)$; por ação vasodilatadora (64).

Os agentes anestésicos, de ação geral e local, modificam o metabolismo energético das células neuronais $(36,39,40)$ e interferem com a estrutura de suas membranas bem como das células em geral (65). Dependendo do tipo de modificações produzidas, esses agentes podem proteger ou lesar os neurônios, em condições de isquêmia $(37,38,58,66)$. Todavia, os resultados não são uniformes (42).

A quetamina, um agente anestésico de ação geral, já havia sido usada em nosso laboratório em preparação de retina isquêmica conferindo significativa neuroproteção seletiva neste tecido $(44,45)$. A escassez de publicações pertinentes (43) induziunos a pesquisar sua ação em modelo de isquemia medular.

A quetamina atua como antagonista não competitivo dos receptores NMDA, impedindo, dessa maneira, ação deletéria dos AAE liberados exageradamente, em condições de isquemia $(44,67)$, (Figuras 2 e 3). Diversamente, a hipotermia parece reduzir a liberação dos AAE no espaço extracelular (68).

Embora estatisticamente a ação comparativa da quetamina e da clorpromazina não tenha al- 
cançado diferença significante, clinicamente a segunda e a associação de ambas parecem mais efetivas.

Não está ainda esclarecida a razão pela qual a ação protetora dessas drogas não atinge todos os animais, considerando o fato de que são isentos de lesão aórtica e que, após a abertura do garrote, a reperfusão deve ser igual em todos. Pode-se especular, todavia, com variações anatômicas da circulação arterial da medula espinal, indentificadas no ser humano $(15-17,69)$ e previsíveis no rato $(70)$.

Destaca-se o fato de que a clorpromazina protegeu $50 \%$ dos ratos quando administrada 1 minuto previamente à isquemia e apenas $10 \%$, quando administrada imediatamente antes da reperfusão. Esta constatação encontra perfeita explicação nas Figuras 2 e 3 , nas quais se observa a máxima elevação dos AAE no período de isquemia. Concluise, daí, que a neuroproteção farmacológica, para ser efetiva, deva ser instituída antes da agressão derivada da isquemia.

Os resultados clínicos do Grupo 4 foram melhores que os do Grupo 3. Pode-se inferir que a quetamina, administrada 10 minutos previamente à isquemia, tenha sido mais eficaz do que quando administrada imediatamente antes.

Pelos resultados desta pesquisa e dos poucos da literatura, a quetamina e a clorpromazina, de uso corrente no ser humano, poderiam ser recomendadas como medida adjuvante na proteção da medula espinal, quando administradas previamente à isquemia deliberada e programada, como acontece na resseção dos aneurismas da aorta. Contudo, há que se tomar todas as cautelas quando se trata de transpor para a clínica, os resultados da pesquisa em animais de laboratório.

A quetamina, elevando a pressão liquórica (18), pode reduzir o fluxo arterial da medula espinal, sendo, portanto, necessário neutralizar esse efeito secundário indesejável.

A clorpromazina, por sua ação vasomotora central e periférica ${ }^{(64)}$, Qcasiona redução da resistência periférica e, conseqũentemente, da pressão arterial. Essas alteração hemodinâmicas, dose-dependentes, são benéficas, em graus moderados, na medida em que aliviam a hipertensão arterial proximal, nos casos de pinçamento aórtico. O fluxo arterial sistêmico pode aumentar, dependendo da importância relativa das mencionadas alterações.

Quaisquer que sejam as medidas profiláticas usadas, elas só terão êxito se, no final do período de isquemia, a perfusão arterial puder ser restabelecida adequadamente. A ressecção dos aneurismas da aorta torácica descendente e da transição toracoabdominal implica em reimplantar ramos importantes que contribuem para a irrigação da medula espinal $(71,72)$. Isso nem sempre é factível e há que se considerar as já mencionadas variações anatômicas.

\section{CONCLUSÃO}

No modelo experimental usado, a quetamina e a clorpromazina, injetadas previamente por via endovenosa, foram eficazes na proteção da medula espinal de ratos, submetida a isquemia de 30 minutos, seguida de reperfusão. 


\section{RBCCV 44205-296}

Sader A A, Coutinho Netto J, Lachat J J, Roselino J E S, Ballerini F J - Pharmacological protection of the ischemic spinal cord. Rev Bras Cir Cardiovasc 1996; 11 (2): 96-106.

ABSTRACT: Ketamine ( $30 \mathrm{mg} / \mathrm{kg}$, IV) and chlorpromazine $(2 \mathrm{mg} / \mathrm{kg}$, IV) were evaluated as pharmacological agents, for protection of the ischemic spinal cord in a rat model. A thirty-minute period of ischemia obtained by occlusion of the proximal descending thoracic aorta was followed by reperfusion. In 70 animals (medium weght- $380 \mathrm{~g}$ ) distributed in 7 equal groups, the results regarding the complete motor and sensitivity function recovery were as follows: 1) Sham-operation: $100 \% ; 2$ ) Ischemia-reperfusion: $0 \%$; 3) Ketamine, 1 minute before ischemia: $30 \%$; 4 ) Ketamine, 10 minutes before ischemia: $50 \%$; 5 Chlorpromazine, 1 minute before isquemia: $50 \% ; 6$ ) Chlorpromazine, 1 minute before reperfusion: $10 \% ; 7)$ Ketamine+chlorpromazine, 1 minute before ischemia: $60 \%$. Both pharmacological agents were effective in the protection of the ischemic spinal cord, as confirmed by the microscopic study. However, comparison of several groups showed statistical significant difference for groups 6 and 7 , only. Perfusion of the subarachnoid space revealed excessive amounts of neuro-excitatories amino-acids, L-aspartate and L-glutamate.

DESCRIPTORS: Spinal cord, drug effects. Ischemia, physiopathology. Ketamine, pharmacology. Chlorpromazine, pharmacology. Spinal cord, ischemic

AGRADECIMENTO: Nossos agradecimentos ao Sr. Sebastião de Assiz Mazzetto, pela colaboração técnica.

\section{REFERÊNCIAS BIBLIOGRÁFICAS}

1 Bigelow W G, Callagham J C, Hopps J A - General hypothermia for experimental intracardiac surgery. Ann Surg 1950; 132: 531-9.

2 Coles J G, Wilson G J, Sima A F et al. - Intraoperative management of thoracic aortic aneurysm: experimental evaluation of perfusion cooling of the spinal cord. J Thorac Cardiovasc Surg 1983; 85: 292-9.

3 Colon R, Frazier O H, Cooley D A, McAllister H A Hypothermic regional perfusion for protection of the spinal cord during periods of ischemia. Ann Thorac Surg 1987; 43: 639-43.

4 Cookson B A, Neptume W B, Bailey C P - Hypothermia as a mean of performing intracardiac surgery under direct vision. Dis Chest 1952; 22: 245-60.

5 LEWIS F J \& TAUFIC M - Closure of atrial septal defects with aid of hypothermia: experimental accoplishments and report of one successful case. Surgery 1953; 33: 52-9.

6 Marsala M, Vanicki I, Galik J, Radonak J, Kundrat I, Marsala J - Panmyelic epidural cooling protects against ischemic spinal cord damage. J Surg Res 1993; 55: 21-31.

7 Pontius R G, Brockman H L, Hardy E G, Cooley D A, DeBakey M E - The use of hypothermia in the prevention of paraplegia following temporary aortic occlusion: experimental observations. Surgery 1954; 36: 33-8.
8 Tabayashi K, Niibori K, Konno H, Mohri H - Protection from postischemic spinal cord injury by perfusion cooling of the epidural space. Ann Thorac Surg 1993; 56: 494-8.

9 Vanicki J, Marsala M, Galik J, Marsala J - Epidural perfusion cooling protection against protracted spinal cord ischemia in rabbits. J Neurosurg 1993; 79: 736-41.

10 Elmore J R, Gloviczki P, Harper Jr. CM et al. - Spinal cord injury in experimental thoracic aortic occlusion: investigation of combined methods of protection. $J$ Vasc Surg 1992; 15: 789-99.

11 Gibbon Jr. J H - Application of a mechanical heart and lung apparatus to cardiac surgery. Minnesota Med $1954 ; 37: 171-80$.

12 Lillehei C W, Cohen M, Warden HE, Zeigler N R, Varco $R L$ - The results of direct vision closure of ventricular septal defects in eight patients by means of controlled cross circulation. Surg Gynecol Obstet 1955; 10: 447-66.

13 Maeda S, Miyamoto $\mathrm{T}$, Murata $\mathrm{H}$ et al. - Prevention of spinal cord ischemia after cross-clamping of the thoracic aorta: monitoring of spinal cord perfusion pressure and somatosensory evoked potencials. Nippon Kyobu Geka Gakkai Zasshi 1989; 37: 1923-31.

14 Schittek A, Bennink G B W E, Cooley D A, Langford L A - Spinal cord protection with intravenous nimodipine: a functional and morphologic evaluation. J Thorac Cardiovasc Surg 1992; 104: 1100.

15 Brewer L A, Fosburg R G, Mulder G A, Verska J J Spinal cord complications following surgery for coarctation of the aorta. J Thorac Cardiovasc Surg 1972; 64: 368-81.

16 Eiseman B \& Summers W B - Factors affecting spina 
Sader A A, Coutinho Netto J, Lachat J J, Roselino J ES, Ballerini FJ - Proteção farmacológica da medula espinal isquêmica. Rev Bras Cir Cardiovasc 1996; 11 (2): 96-106.

cord ischemia during aortic occlusion. Surgery 1955 ; 38: $1063-70$.

17 Fereshetian A, Saadoon K, Kaufman S L et al. - Digital subtraction spinal cord angiography in patients undergoing thoracic aneurysm surgery. Cardiovasc Intervent Radiol 1989; 12: 7-9.

18 Shapiro H M, Wyte S R, Harris A B - Ketamine anaesthesia in patients with intracranial pathology. Br J Anaesth 1972; 44: 1200-4.

19 Blaisdell F W \& Cooley D A - The mechanism of paraplegia after temporary thoracic aortic occlusion and its relationship to spinal fluid pressure. Surgery 1962; 51: 351-5.

20 Francel P C, Long B A, Malik J M, Tribble C, Jane J A, Kron I L - Limiting ischemic spinal cord injury using a free radical scavenger 21-aminosteroid and/or cerebrospinal fluid drainage. J Neurosurg 1993; 79: 742-51.

21 McCullough J L, Hollier L H, Nugent M - Paraplegia after thoracic aortic occlusion. Influence of cerebrospinal fluid drainage: experimental and early clinical results. J Vasc Surg 1988; 7: 153-60.

Woloszyn T T, Marini C P, Coons M S et al. Cerebrospinal fluid drainage and steroids provide better spinal cord protection during aortic crossclamping than does either treatment alone. Ann Thorac Surg 1990; 49: 78-83.

Johnson S H, Kraimer J M, Graeber G M - Effects of flunarizine on neurological recovery and spinal cord blood flow in experimental spinal cord ischemia in rabbits. Stroke 1993; 24: 1547-53.

24 Lyden P D, Zivin J A, Kochhar A, Mazzerella V - Effects of calcium channel blockers on neurologic outcome after focal ischemia in rabbits. Stroke 1988; 19: 1020-6.

25 Madden K P. Clark W M, Marcoux F W et al. - Treatment with conotoxin, an $\mathrm{N}$-type calcium channel blocker, in neuronal hypoxic - ischemic injury. Brain Res 1990; 537: 256-62.

26 Svensson L G, Von Ritter C M, Groeneveld H T, Rickards E S - Cross-clamping of the thoracic aorta. Influence of aortic shunts, laminectomy, papaverine, calcium channel blocker, allopurinol, and superoxide dismutase on spinal cord blood flow and paraplegia in baboons. Ann Surg 1986; 204: 38-47.

27 Agee J M, Flanagan T, Blackbourne L H, Kron I L, Tribble C G - Reducing postischemic paraplegia using conjugated superoxide dismutase. Ann Thorac Surg 1991; 51: $911-5$.

28 Cuevas P, Reimers D, Carceller F, Jimenez A - Ischemic reperfusion injury in the rabbit spinal cord: protective effect of superoxide dismutase on neurological recovery and spinal infarction. Acta Anat 1990; 137: 303-10.

29 Kirshner D L, Kirshner R L, Heggeness L M, DeWeese $J$ A - Spinal cord ischemia: an evaluation of pharmacologic agents in minimizing paraplegia after aortic occlusion. J Vasc Surg 1989; 9: 305-8.

30 Qayumi A K, Janusz M T, Jamieson W R E, Lyster D $M$ - Pharmacologic interventions for prevention of spinal cord injury caused by aortic crossclamping. $J$ Thorac Cardiovasc Surg 1992; 104: 256-61.

31 Clark W M, Madden K P, Rothlein R, Zivin J A - Reduction of central nervous system ischemic injury in rabbits using leukocyte adhesion antibody treatment. Stroke 1991; 22: 877-83.

32 Clark W M, Madden K P, Rothlein R, Zivin J A - Reduction of central nervous system ischemic injury by monoclonal antibody to intercellular adhesion molecule. I Neurosurg 1991; 75: 623-7.

33 Kocchar A, Zivin J A, Lyden P D, Mazzarela V Glutamate antagonist therapy reduces neurologic deficits produced by focal central nervous system ischemia. Arch Neurol 1988; 45: 148-53.

34 Madden K P, Clark W M, Kochhar A, Zivin J A -Efficacy of LY233053, a competitive glutamate antagonist, in experimental central nervous system ischemia. $J$ Neurosurg 1992; 76: 106-110.

35 Martinez-Aarizala A, Rigamont D D, Long J B, Kraimer $\mathrm{J} \mathrm{M}$, Holaday $\mathrm{J} \mathrm{W}$ - Effects of NMDA receptor antagonists following spinal ischemia in the rabbit. Exp Neurol 1990; 108: 232-40.

36 Astrup J, Sörensen P M, Sörensen H R - Inhibition of cerebral oxygen and glucose consumption in the dog by hypothermia, pentobarbital, and lidocaine. Anesthesiology 1981; 55: 263-8.

37 Cavazzuti M, Porro C A, Barbieri A, Galetti A -Brain and spinal cord metabolic activity during propofol aniaesthesia. Brit J Anaesth 1991; 66: 490-5

38 Frawley J E, Hicks R G, Horton D A, Gray I J, Niesche J W, Matheson J M - Thiopental sodium cerebral protection during carotid endarterectomy: perioperative disease and death. J Vasc Surg 1994; 19: $732-8$.

39 Geddes I C \& Quastel J H - Effects of local anaesthetics on respiration of rat brain cortex in vitro. Anesthesiology 1956; 17: 666-71.

40 Haschke R $H$ \& Fink B R - Lidocaine effects on brain mitochondrial metabolism in vitro. Anesthesiology 1975; 42: 737-40.

41 Kochs E, Hoffman W E, Werner C, Thomas C, Albrecht $R$ F, Esch J S - The effects of propofol on brain electrical activity, neurologic outcome, and neuronal damage following incomplete ischemia in rats. Anesthesiology 1992; 76: 245-52. 
Sader A A, Coutinho Netto J, Lachat J J, Roselino J ES, Ballerini FJ - Proteção farmacológica da medula espinal isquêmica. Rev Bras Cir Cardiovasc 1996; 11 (2): 96-106.

Michenfelder J D - The interdependency of cerebral functional and metabolic effects following massive doses of thiopental in the dog. Anesthesiology 1974; 41: $231-6$

43 Naslund T C, Hollier L H, Money S R, Facundus E C, Skenderis II B S - Protecting the ischemic spinal cord during aortic clamping: the influence of anesthetics and hypothermia. Ann Surg 1992; 215: 409-16.

44 Louzada Júnior P, Dias J J, Santos W F, Bradford H F, Lachat $\mathrm{J} \mathrm{J}$, Coutinho Netto $\mathrm{J}$ - Glutamate release in experimental ischemia of the retina: an aproach using microdialysis. J Neurochem 1992; 59:358-63.

Santos W A - Aminoácidos excitatórios na lesão neural seletiva por isquemia seguida de reperfusão. Papel de antagonistas na proteção contra o processo isquêmico. [Dissertação. Mestrado] Ribeirão Preto, SP: Faculdade de Medicina de Ribeirão Preto, Universidade de São Paulo, 1995. 97p.

Chien K R, Abrams J, Pfau R G, Farber J L - Prevention by chlorpromazine of ischemic liver cell death. $A m$ J Pathol 1977; 88: 539-58.

47 Roselino J E S, Castro-E-Silva Jr. O, Romanello L M F, Ceneviva R - Effect of chlorpromazine and biliary drainage on portal blood flow and mitochondrial function during extrahepatic cholestasis. Brazilian $J$ Med Biol Res 1989; 22: 889-93.

48 Edoute Y, Van Der Merve E L, Sanan D, Kotze J C N, Van Niekeerke I, Lochner A - Normothermic ischaemic cardiac arrest and reperfusion of the isolated working heart: effect of chlorpromazine on functional metabolic and morphological recovery. J Mol Cell Cardiol 1983; 15: 603-20.

Kruel N F - Desenvolvimento de modelo experimental para comparar aspectos metabólicos do miocárdio de coelhos submetidos à cardioplegia. [Tese. Doutorado] Ribeirão Preto, SP: Faculdade de Medicina de Ribeirão Preto, Universidade de São Paulo, 1987.

50 Thomas G E, Levistsky S, Feinberg H - Chlorpromazine inhibits loss of contractive function, compliance and ATP in ischemic rabbit heart. $J \mathrm{Mol}$ Cell Cardiol 1983; 15: 621-28.

51 Balestrino M \& Somjen G G - Chlorpromazine protects brain tissue in hypoxia by delaying spreading depression-mediated calcium influx. Brain Res 1986; 385: 219-26.

52 Zivin J A, Kochhar A, Saitoh T - Phenothiazines reduce ischemic damage to the central nervous system. Brain Res 1989; 482: 189-93.

53 Lindroth $P$ \& Mopper K - High performance liquid chromatography of subpicomole amounts of amino acids by pre colum fluorescence pre derivatization with o-phthalaldehyde. Ann Chem 1979; 51: 1667-777.
54 Borst H G, Jurmann M, Buhner B, Laas J - Risk of replacement of descending aorta with a standardized left heart bypass technique. J Thorac Cardiovasc Surg 1994; 107: 126-32.

55 Murad H, Albuquerque P C C, Piedade M M - Isquemia medular por deprivação sangüinea temporária: estudo experimental em cães. Rev Col Bras Cir 1981; 8: $78-83$.

56 Schepens M A, Defauw J J, Hamerlijnck R P, De Geest $R$, Vermeulen F E - Surgical treatment of thoracoabdominal aortic aneurysms by simple crossclamping: risk factors and late results. $J$ Thorac Cardiovasc Surg 1994; 107: 134-42.

57 Siesjö B K - Calcium and ischemic brain damage. Eur Neurol 1986; 25 (Suppl 1): 45-56.

58 Kocchar A, Saitoh T, Zivin J - Reduced protein kinase C activity in ischemic spinal cord. I Neurochem 1989; 53: 946-52.

59 Burda J \& Chavko M - Effect of ischaemia on protein synthesis in neuron and glia-enriched fractions from the rabbit spinal cord. Physiol Res 1991; 41: 39-47.

60 Bulkley G B - Reactive oxygen metabolites and reperfusion injury: aberrant triggering of reticuloendothelial function. Lancet 1994; 344: 934-6.

61 Rothaman S M \& Olney J W - Glutamate and the pathophysiology of hipoxic-ischemic brain damage. Ann Neurol 1986; 19: 105-11.

62 Yum S W \& Faden A I - Comparison of neuroprotective effects of the $\mathrm{N}$-methyl - D - aspartate antagonist MK- 801 and the opiate-receptor antagonist nalmefene in experimental cord ischemia. Arch Neurol 1990; 47: $277-81$

63 Juskevich J C, Kuhn D M, Lovenberg W - Phosphorylation of brain cystosol proteins. J Biol Chem 1983; 258: 1950-53.

64 Queiroz O A - Estudo de variáveis hemodinâmicas, da temperatura do halux e do potencial hidrogeniônico muscular com o uso de benciclam, nitroprussato de sódio e clorpromazina em pacientes submetidos a cirurgia cardiaca com circulação extracorpórea [Tese. Doutorado] Ribeirāo Preto, SP: Faculdade de Medicina de Ribeirăo Preto, Universidade de São Paulo, 1989.

65 Seeman P - Pharmacol Rev 1972; 24: 583-655.

66 Breckwoldt W L, Genco C M, Conolly R J, Cleveland R $\mathrm{J}$, Diehl J T - Spinal cord protection during aortic occlusion: efficacy of intrathecal tetracaine. Ann Thorac Surg 1991; 51: 959-63.

67 Benveniste H, Drejer J, Schousboue A, Diemer N H. Elevation of the extracelular concentration of gluatamate in rat hippocampus during transient ischemic monitored by intracerebral microdialysis. $J$ Neurochem 1984; 43; 1369-74. 
Sader A A, Coutinho Netto J, Lachat J J, Roselino J ES, Ballerini F J - Proteção farmacológica da medula espinal isquêmica. Rev Bras Cir Cardiovasc 1996; 11 (2): 96-106.

68 Rokkas C K, Cronin C S, Nitta T et al. - Profound systemic hypothermia inhibits the release of neurotransmitter amino acids in spinal cord ischemia. J Thorac Cardiovasc Surg 1995; 110: 27-35.

69 Wadouh F, Lindemann E M, Arndt C F, Hetzer R, Borst $\mathrm{H} \mathrm{G}$ - The arteria radicularis magna anterior as a decisive factor influencing spinal cord damage during aortic occlussion. J Thorac Cardiovasc Surg 1984; 88: 1-10.

\section{Discussão}

\section{PROF. OTONI MOREIRA GOMES Belo Horizonte, MG}

Agradeço à Comissão Organizadora do nosso Congresso a imensa honra desta participação e, de modo especial, pela seleção desta excelente investigação para nossos comentários. Presto ao Professor Albert Sader e seus insignes colaboradores uma singela, porém sincera homenagem em nome de toda nossa equipe e do Corpo Docente do Curso de Pós-Graduação em Cirurgia Cardiovascular do Serviço do Coração da Fundação Cardiovascular São Francisco de Assis, entusiasmados com a linha de pesquisa aqui aberta, com a importância do tema escolhido, com a objetividade do método e da interpretação dos resultados obtidos. Em passado recente, a constatação contundente de complicações neurológicas severas após cirurgias na aorta torácica tinha, como lenitivo para a equipe cirúrgica, a compreensão do paciente e de seus familiares, frente à evidência do risco imposta pela própria lesão. Hoje, e com mais certeza nos próximos anos, face à divulgação dos progressos tecnológicos pela mídia maciça, onde só o ótimo é ensinado, a compreensão social na paraplegia pós-operatória tende a diminuir rapidamente e ser substituida pela agressividade jurídica, aumentando o sofrimento da equipe cirúrgica já consternada pela impotência do estado de técnica na sua prevenção. A importância da linha de pesquisa apresentada é também evidente em inúmeras pesquisas internacionais recentes como as de Rokkas e colaboradores; em 1994, demonstrando proteção medular com infusão venosa de Dextrorphano; a de Szemptpetery, Crister e Grinnan, em 1993, afirmando que, embora estudos experimentais demonstrem que a hipotermia ofereça proteção medular durante isquemia de até 45 minutos, a paraplegia ainda permanece como maior compli-
70 Schievink W I, Luyendijk W, Los J A - Does the artery of Adamkiewicz exist in the albino rat? J Anat 1988; 161: 95-101.

71 Gray, ed. Warnrick R \& Willians P L, trads. Gray Anatomia. 35. ed. Rio de Janeiro: Guanabara Koogan, 1979: vol. 2, p. 795.

Ranson, S W - Anatomia do sistema nervoso central. 7. ed. São Paulo: Renascença SA, 1943: p. 104-5.

cação da operação na aorta torácica descendente, e a Nicolosi e colaboradores, publicada no Annals of Thoracic Surgery neste mês de março, mostrando incidência de até $17 \%$ de paraplegia no tratamento da rotura traumática da aorta, operada sem circulação extracorpórea. Na pesquisa aqui apresentada, o Professor Sader e seus colaboradores, utilizando drogas já aprovadas e amplamente testadas em uso clínico geral, oferece uma alternativa de rápida aplicação clínica. A avaliação dos resultados não poderia ser mais objetiva, bem a gosto dos cirurgiões. Creio que o Prof. Waldyr Jazbik, com a objetividade e perspicácia de seu tirocínio, que muitos de nós já conhecem, assim resumiria estas conclusões: "andou, está bom; não andou, não presta". Parabéns, Professor Sader. Muitos animais andaram com uso de sua técnica. A pesquisa, por sua originalidade, pelo seu interesse clínico e pelo potencial de aplicação cirúrgica, é realmente excelente.

\section{PROF. SADER \\ (Encerrando)}

Meus agradecimentos ao Prof. Otoni Moreira Gomes, pelos comentários generosos e estimulantes. O trabalho apresentado não representa a solução definitiva do problema da isquemia medular. Creio, todavia, que seja um passo importante nessa linha de pesquisa que estamos iniciando. Como, em todas as pesquisas realizadas em animais de laboratório, a transposição de seus resultados para o ser humano deve ser feita com cautela e dentro dos critérios de segurança. Sempre que possível, recomendo que este recurso seja usado em adição e não em substituição aos demais, já em uso. Obviamente, qualquer método de proteção à medula espinal isquêmica só será efetivo se, no final do período de isquemia, a circulação sangüínea arterial for restabelecida a fluxos adequados. 